\title{
Avaliação da Anastomose de Artéria Torácica Interna Esquerda com Artéria Interventricular Anterior pela Ecodopplercardiografia
}

\author{
A delaide A rruda, 0 rlando Campos Fo, Expedito Ribeiro, A ntônia Petrizzo, José Lázaro Andrade, \\ Antônio Carlos C. Carvalho, Enio Buffolo, Eulógio Martinez, W ilson Mathias Jr
}

São Paulo, SP

Objetivo - Avaliar o valor do ecocardiograma Doppler(ECO) transtorácico na identificação de perviabilidade da anastomose entre artéria torácica interna esquerda (ATIE) e interventricular anterior, realizada pela técnica de revascularização miocárdica pela minitoracotomia sem circulação extracorpórea.

Métodos - Estudaram-se os primeiros 12 pacientes, consecutivos, no período de pós-operatório intra-hospitalar pelo ECO, utilizando-se transdutores de $5 \mathrm{MHz}$, pela via paraesternal esquerda, preferencialmente. Foram analisadas velocidades máximas e integrais de velocidade dos componentes sistólico e diastólico das curvas espectrais de fluxo Doppler. Todos pacientes foram submetidos à cinecoronariografia, enquanto hospitalizados.

Resultados - O ECO foi exeqüivel em $93 \%$ dos pacientes. Nos com anastomose pérvia (6/7), observou-se ao estudo Doppler amplo componente diastólico (padrão A). $\mathrm{Na}$ queles com anastomose obstruída (4/4) o padrão observado foi de predomínio sistólico (padrão $B)(p=0,003 *)$.

Conclusão - O ECO da ATIE anastomosada com a artéria interventricular anterior, após cirurgia de revascularização miocárdica pela técnica de minitoracotomia, permitiu caracterizar precocemente, com precisão, a perviabilidade da anastomose.

Palavras-chave: ecocardiografia, artérias torácicas, revascularização

\section{Evaluation of Left Internal Thoracic Artery Anastomosis with Left Anterior Descending Coronary Artery by Doppler Echocardiography}

Purpose - To study the value of Doppler echocardiography as a tool for the evaluation of left internal thoracic artery graft (LITAG) patency in patients who underwent coronary revascularization using minimally invasive bypass surgery without extracorporeal circulation.

Methods - The first 12 consecutive patients were studied after coronary artery bypass surgery using a $5 \mathrm{MHz}$ Doppler transducer. Doppler signals for the systolic and diastolic flow velocities were preferably obtained in the second intercostal space. All patients underwent coronary angiography while hospitalized.

Results - The exam was feasible in 93\% of patients. Doppler flow pattern was predominantly diastolic (pattern A) in patients with patent anastomoses (6/7). In patients with occluded anastomoses (4/4) Doppler flow pattern was predominantly systolic (pattern $B)(p=0.003)$.

Conclusion - Internal thoracic artery flow pattern as measured by Doppler echocardiography after minimally invasive coronary artery bypass graft surgery is an accurate method for identifying LITAG patency.

Key-words: Doppler echocardiography; thoracic artery; bypass surgery

Arq Bras Cardiol, volume 69 (nº 6), 413-419, 1997

Durante as últimas décadas, vem sendo observada em países desenvolvidos, sensível queda nas taxas de mortalidade por coronariopatia aterosclerótica, fato que pode es-

Escola Paulista de Medicina-UNIFESP e Hospital Unicor - São Paulo Correspondência: Adelaide Maria Martins Arruda - Rua Itapeva, 500 - conj 5C 01332-000 - São Paulo, SP

Recebido para publicação em 7/8/97

Aceito em 16/10/97 tar relacionado ao sucesso de medidas preventivas e terapêuticas na abordagem desses pacientes ${ }^{1,2}$. No manuseio terapêutico atual de pacientes com coronariopatia aterosclerótica, vários aspectos são considerados: correção dos fatores de risco, mudanças no estilo de vida, emprego de fármacos e, por fim, a revascularização miocárdica, seja por angioplastia transluminal coronária, seja por cirurgia.

Atualmente, a artéria torácica interna é considerada o 
enxerto de primeira escolha para a cirurgia de revascularização miocárdica, por ser menos vulnerável ao desenvolvimento de doença aterosclerótica ${ }^{3}$.

A cirurgia de revascularização miocárdica, geralmente, é realizada através de esternotomia mediana, com emprego de circulação extracorpórea (CEC), cardioplegia e hipotermia. Como opção cirúrgica, alguns centros médicos realizam a revascularização miocárdica sem CEC. Esta técnica encontra-se consolidada pela grande experiência acumulada em centros médicos específicos e apresenta tendência crescente a ser incorporada por outros grupos ${ }^{4,5}$.

O refinamento dos procedimentos operatórios têm permitido vias de acesso alternativas com dimensões reduzidas, denominadas cirurgias através de mínima incisão torácica. A técnica pode ser utilizada com ou sem auxílio de CEC percutânea, sob visão direta ou com uso de toracoscopia $^{6}$. Como a cirurgia de revascularização miocárdica por minitoracotomia é de emprego recente e, portanto, está em aperfeiçoamento, a experiência atual dos grupos que a utilizam é inicial é bastante limitada ${ }^{7,8}$. Em nosso meio, a revascularização miocárdica por minitoracotomia tem sido realizada com a confecção das anastomoses sob visão direta, sem CEC, com experiência crescente ${ }^{9,10}$.

Considerando-se que a cirurgia de revascularização miocárdica pela minitoracotomia constitui uma técnica em expansão, torna-se necessária a utilização de um método que avalie seus resultados imediatos e tardios, preferencialmente de forma não invasiva.

O objetivo deste estudo foi avaliar o valor do ecocardiograma Doppler(ECO) transtorácico para o diagnóstico de perviabilidade da anastomose da artéria torácica interna esquerda (ATIE) com a interventricular anterior, no período de pós-operatório (PO) intra-hospitalar de pacientes submetidos à cirurgia de revascularização miocárdica pela técnica de minitoracotomia sem CEC, utilizando-se a cinecoronariografia (CINE) como padrão de referência.

\section{Métodos}

De setembro/95 a agosto/96, a equipe de Cirurgia Cardiovascular da Universidade Federal de São Paulo - Escola Paulista de Medicina realizou em três diferentes instituições hospitalares 45 intervenções cirúrgicas de revascularização miocárdica pela técnica de minitoracotomia sem CEC. Desta casuística, derivaram os pacientes do presente estudo, entre aqueles operados nos Hospitais São Paulo e Unicor, selecionados conforme os seguintes critérios: pacientes com estenose $\geq 70 \%$ do diâmetro da luz da coronária interventricular anterior à CINE, com angina do peito ou infarto do miocárdio, submetidos a revascularização miocárdica por minitoracotomia sem $\mathrm{CEC}^{10}$; pacientes com ECO e CINE realizados no período PO intra-hospitalar com intervalo de tempo entre a cirurgia e os exames inferior a 30 dias e com intervalo entre os exames não superior a 12 dias. Foram excluídos os pacientes que não possuíam CINE e estudo ecocardiográfico, ou com infecções da ferida cirúrgica, derrame pleural ou arritmias cardíacas graves. Inicialmente, nos 12 pacientes consecutivos que corresponderam à experiência inicial da equipe de Cirurgia Cardiovascular, não foi possível obter o fluxo Doppler, tanto pela via paraesternal quanto pela supraclavicular, em apenas um paciente, por janela acústica inadequada. O estudo ECO foi, portanto, exeqüível em 11, ou seja, 93\% da amostra inicial. Destes, sete eram do sexo masculino e quatro do feminino. A idade variou de 42 a 83 (média de 57) anos. As características clínicas e angiográficas prévias à intervenção cirúrgica encontram-se na tabela I. A técnica cirúrgica utilizada foi a preconizada por Teles e col ${ }^{10}$.

No período PO, a anastomose entre as artérias torácica interna e interventricular anterior era avaliada, subjetivamente, pela CINE de controle, como sendo pérvia ou obstruída, dando origem a dois grupos: grupo I - pacientes com anastomose pérvia e grupo II - pacientes com anastomose obstruída (ausência de fluxo angiográfico através da anastomose).

Como grupo controle foram estudados 12 indivíduos, hígidos, com idade variando entre 26 a 40 (média de 30) anos, sem antecedentes mórbidos, considerados como grupo controle. Todos apresentavam exame clínico, eletrocardiograma de 12 derivações e ECO transtorácico normais. Os exames foram de fácil realização com curvas espectrais de fluxo sangüíneo obtidas pelo estudo Doppler de boa qualidade. Não houve exclusão de indivíduos do grupo controle por janela acústica inadequada.

A imagem da ATIE e o respectivo estudo Doppler do seu fluxo sangüíneo foram realizados em aparelhos da marca Advanced Tecnology Laboratories (ATL) dos seguintes modelos: Ultramark 9 HDI, Ultramark 9 Digital plus e Ultramark 4 plus, equipados com transdutores eletrônicos ou mecânicos de $5 \mathrm{MHz}$ com Doppler pulsátil dirigido pelas imagens bidimensionais. Os ECO foram obtidos com a mesma aparelhagem. As curvas espectrais de velocidade do fluxo sangüíneo da ATIE, obtidas pelo estudo Doppler, foram gravadas, por aparelhos de videocassete da marca Panasonic acoplados aos ecocardiógrafos e utilizadas para posterior análise. Para este fim, usou-se equipamento dotado de sistema de imagens digitais denominado image view, fabricado pela Novamicrosonics para mensurações posteriores.

Os pacientes e os indivíduos do grupo controle foram, inicialmente, submetidos a ECO transtorácico em decúbito lateral esquerdo. Após a realização do estudo convencional, procedeu-se a avaliação do fluxo da porção proximal da ATIE, com o paciente em decúbito dorsal horizontal. O $2^{\circ}$ espaço intercostal esquerdo paraesternal foi considerado o local preferencial para abordagem desta estrutura. A via supraclavicular esquerda ${ }^{11}$ poderia ser utilizada como alternativa em pacientes cuja janela acústica intercostal fosse inadequada para obter curvas de qualidade satisfatória. Os exames foram realizados por um único observador, com monitorização eletrocardiográfica contínua de uma derivação isolada.

Pela via paraesternal esquerda, a ATIE era identificada através do estudo bidimensional, caracterizada como estru- 
Tabela I - Características clínicas e angiográficas pré-operatórias dos pacientes submetidos à revascularização miocárdica pela minitoracotomia sem circulação extracorpórea

\begin{tabular}{|c|c|c|c|c|c|c|c|}
\hline Pts & Sx & Id & $\begin{array}{c}\text { Doenças } \\
\text { Associadas }\end{array}$ & $\begin{array}{l}\text { Diagnóstico } \\
\text { Cardiológico }\end{array}$ & $\begin{array}{c}\text { Estenoses } \\
\text { coronárias } \geq 70 \%\end{array}$ & Disfunção VE & $\begin{array}{c}\text { Intervenções } \\
\text { Associadas }\end{array}$ \\
\hline 1 & M & 50 & - & $\mathrm{AI}$ & AIVA & aus & - \\
\hline 2 & $\mathrm{M}$ & 58 & HAS & $\mathrm{AE}$ & AIVA & $\bmod$ & - \\
\hline 3 & M & 45 & & $\mathrm{AE}$ & AIVA & aus & - \\
\hline 4 & $\mathrm{~F}$ & 44 & & AI & AIVA & aus & - \\
\hline 5 & M & 42 & HAS & IAM & AIVA & $\bmod$ & - \\
\hline 6 & $\mathrm{~F}$ & 56 & HAS & AI & AIVA, ACD & aus & - \\
\hline 7 & $\mathrm{M}$ & 60 & HAS & $\mathrm{AI}$ & AIVA & aus & - \\
\hline 8 & M & 68 & HAS,DM & IAM & AIVA, ACD & dis & Atc e stent na ACD \\
\hline 9 & $\mathrm{~F}$ & 83 & HAS & $\mathrm{AI}$ & AIVA, ACX & dis & - \\
\hline 10 & $\mathrm{~F}$ & 68 & HAS,DM,IR & AI & AIVA, ACD & aus & \\
\hline 11 & M & 60 & & AI & AIVA, ACX & aus & \\
\hline
\end{tabular}

ACD- artéria coronária direita; ACX- artéria coronária circunflexa; AIVA- artéria interventricular anterior; AE- angina estável; AI- angina instável; ATC- angioplastia transluminal coronária; Aus- ausente; DM- diabetes mellitus; Dis- discreta; F- feminino; HAS- hipertensão arterial sistêmica; IAM- infarto agudo do miocárdio prévio; Id- idade em anos; IR- insuficiência renal; M- masculino; Mod- moderada; Pts- pacientes; Sx- sexo; VE- ventricular esquerda.

tura vascular, tubular e não pulsátil, justaposta à margem esquerda do esterno. A amostra de volume do Doppler pulsátil com dimensões reduzidas (1,5mm a $3 \mathrm{~mm})$ era então posicionada à luz da ATIE, sendo realizadas discretas angulações laterais e craniais do transdutor, de forma a se obter curvas espectrais de fluxo sangüíneo, com as maiores velocidades e menor dispersão de freqüências possíveis. Os ajustes de ganho e filtros foram feitos para se obter as curvas de melhor definição e menor ruído. A correção de ângulo do Doppler foi utilizada em todos os exames.

A figura 1 ilustra, a posição do transdutor, na superfície do tórax, para a obtenção das curvas espectrais de fluxo sangüíneo por meio do ECO pelas vias paraesternal e supraclavicular esquerdas.

A análise das curvas das velocidades de fluxo Doppler da artéria torácica interna foi realizada simultanea-

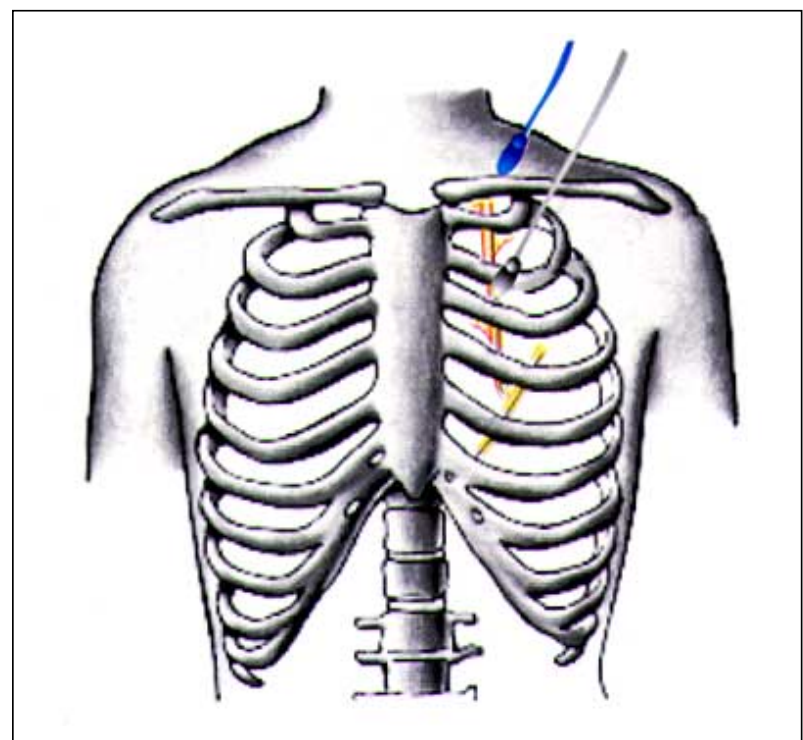

Fig. 1 - Esquema da posição do transdutor para obtenção de curvas espectrais de fluxo sangüíneo da artéria torácica interna esquerda por meio da ecocardiografia Doppler pelas vias paraesternal e supraclavicular esquerdas.

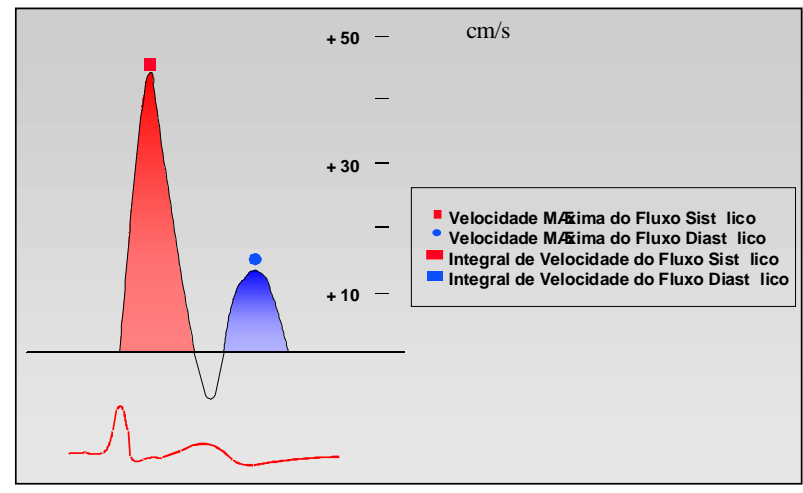

Fig. 2 - Diagrama da curva de fluxo de artéria torácica interna esquerda obtida pelo ecocardiograma Doppler por via paraesternal esquerda em um indivíduo normal.

mente por dois observadores, a partir das gravações em fitas de videoteipe e os seguintes dados do ECO foram analisados nos indivíduos do grupo controle e nos pacientes: a) análise qualitativa dos padrões morfológicos das curvas espectrais de fluxo sangüíneo da ATIE; b) análise quantitativa dos parâmetros das velocidades de curvas espectrais do fluxo sangüíneo da ATIE detalhados a seguir: 1) velocidade máxima do fluxo diastólico (VMD), expressa em $\mathrm{cm} / \mathrm{s}$; 2) velocidade máxima do fluxo sistólico(VMS), expressa em $\mathrm{cm} / \mathrm{s} ; 3$ ) razão entre a velocidade máxima do fluxo diastólico e a velocidade máxima do fluxo sistólico (índice VMD/ VMS); 4) integral de velocidade do fluxo diastólico (IVD) expressa em $\mathrm{cm}$; 5) integral de velocidade do fluxo sistólico (IVS) expressa em cm; 6) razão entre a integral de velocidade do fluxo diastólico e a integral de velocidade do fluxo sistólico (índice IVD/IVS). O diagrama de fluxo de artéria torácica de um indivíduo normal encontra-se na figura $2 ; \mathrm{c}$ ) distribuição de pacientes com índices VMD/VMS e IVD/ IVS >0,5 nos pacientes dos grupos I e II.

Para comparação dos parâmetros obtidos pelo estudo Doppler, bem como da freqüência cardíaca (FC) dos pacientes no grupo I e II e nos indivíduos do grupo controle foi utilizado o teste Kruskal-Wallis. O teste exato de Fisher foi 
empregado para análise da distribuição dos índices VMD/ VMS e IVD/IVS obtidos nos pacientes do grupo I e II. Para a associação entre os padrões de curvas espectrais de fluxo Doppler e as CINE de controle PO foi utilizado o coeficiente de concordância de Kappa. Consideramos $\mathrm{K}<0,40$ baixa reprodutibilidade, $0,40 \leq \mathrm{K} \leq 0,75$ como boa reprodutibilidade e $\mathrm{K}>0,75$ como excelente reprodutibilidade. Em todos os testes, fixou-se em 0,05 ou $5 \%$ (p < $<0,05)$ o nível de rejeição para a hipótese de nulidade, assinalando-se com um asterisco os valores significantes. Os valores não significantes foram expressos por "ns".

\section{Resultados}

As intervenções cirúrgicas transcorreram sem anormalidade em todos os casos. Os pacientes tiveram alta da unidade de terapia intensiva no $1^{\circ}$ dia de PO. No período de PO intra-hospitalar, os pacientes evoluíram de forma estável sem maiores intercorrências clínicas e as CINE de controle PO, também, transcorreram sem maiores intercorrências.

O estudo Ecocardiográfico das artérias torácicas internas foi realizado tanto nos pacientes quanto nos indivíduos do grupo controle, por meio da via preferencial paraesternal esquerda, obtendo-se curvas de boa definição para a análise adequada dos seus componentes sistólico e diastólico.

Em geral, do ponto de vista morfológico, foram obtidos dois tipos característicos de curva: padrão A - curvas espectrais de estudo Ecocardiográfico da ATIE anastomosada à artéria interventricular anterior com aspecto peculiar apresentando predomínio do componente diastólico (fig. 3); padrão B - curvas espectrais do ECO da ATIE anastomosada à interventricular anterior com predomínio do componente sistólico (fig. 4).

\begin{tabular}{|c|c|c|c|}
\hline \multicolumn{4}{|c|}{$\begin{array}{l}\text { Tabela II - Comparação entre os padrões morfológicos das curvas } \\
\text { espectrais de Doppler e resultados da cinecoronariografia do pós- } \\
\text { operatório }\end{array}$} \\
\hline \multirow[t]{2}{*}{ Doppler } & \multicolumn{3}{|c|}{ Cinecoronariografia } \\
\hline & Grupo I & Grupo II & Total \\
\hline Padrão A & 6 & 0 & 6 \\
\hline Padrão B & 1 & 4 & 5 \\
\hline Total & 7 & 4 & 11 \\
\hline \multicolumn{4}{|c|}{ Kappa- 0,$81 ;$ p- $0,0030^{*}$} \\
\hline
\end{tabular}

\begin{tabular}{|lccc|}
\hline \multicolumn{5}{|c|}{ Tabela III - Distribuição dos índices VMD/VMS e IVD/IVS superiores } \\
a 0,5 nos grupos I e II
\end{tabular}

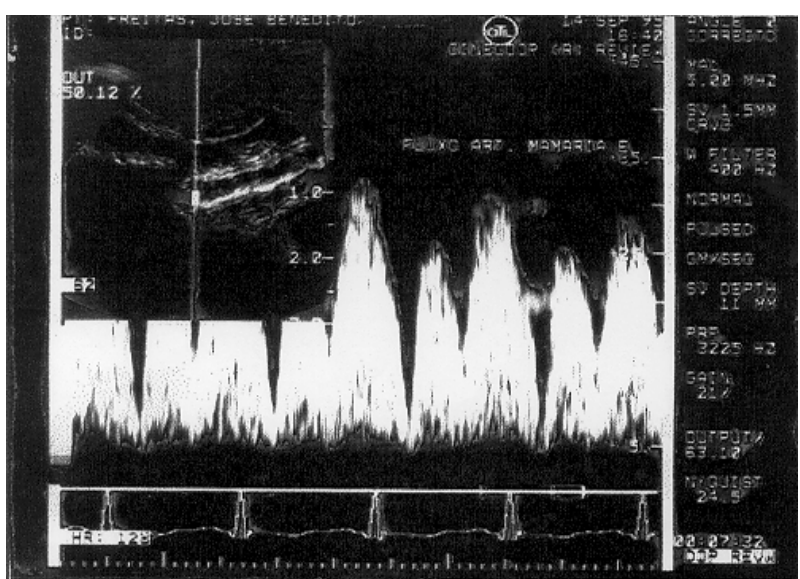

Fig. 3 - Exemplo de curva de fluxo com padrão A, obtida em artéria torácica interna esquerda anastomosada à interventricular anterior, obtida pelo ecodopplercardiograma por via paraesternal esquerda.

Foram confrontados os tipos de curva (padrão A e B) com os resultados da CINE de controle PO (grupo I e II), que expressaram a situação funcional da anastomose (tab. II).

A morfologia das curvas de Doppler, predominando nos pacientes do grupo I, foi o padrão $\mathrm{A}$, enquanto o padrão B, foi mais freqüente, nos pacientes do grupo II.

A análise dos parâmetros do componente sistólico das curvas espectrais de fluxo Doppler, isto é, para a velocidade máxima de fluxo sistólico e integral de velocidade de fluxo sistólico não evidenciou diferença estatística significativa entre os valores obtidos nos grupos I, II e controle.

Observou-se que os valores obtidos para parâmetros do componente diastólico da curva espectral de fluxo Doppler, ou seja, velocidade máxima de fluxo diastólico e a integral de velocidade de fluxo diastólico, nos pacientes do grupo I foram significativamente superiores ao grupo II e controle. Não houve diferença estatística desses parâmetros entre grupos II e controle (fig. 5).

Os valores dos índices VMD/VMS e IVD/IVS foram superiores, do ponto de vista estatístico, no grupo I, quando comparados aos grupos II e controle. Entretanto, não houve diferença deste índice entre os grupos II e controle (fig.6).

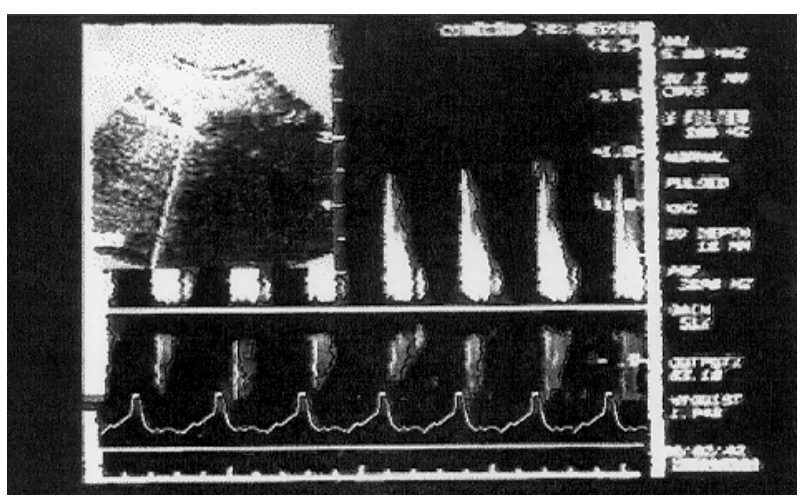

Fig. 4 - Exemplo de curva de fluxo com padrão B, obtida em artéria torácica interna esquerda anastomosada à interventricular anterior, pelo ecocardiograma Doppler por via paraesternal esquerda. 


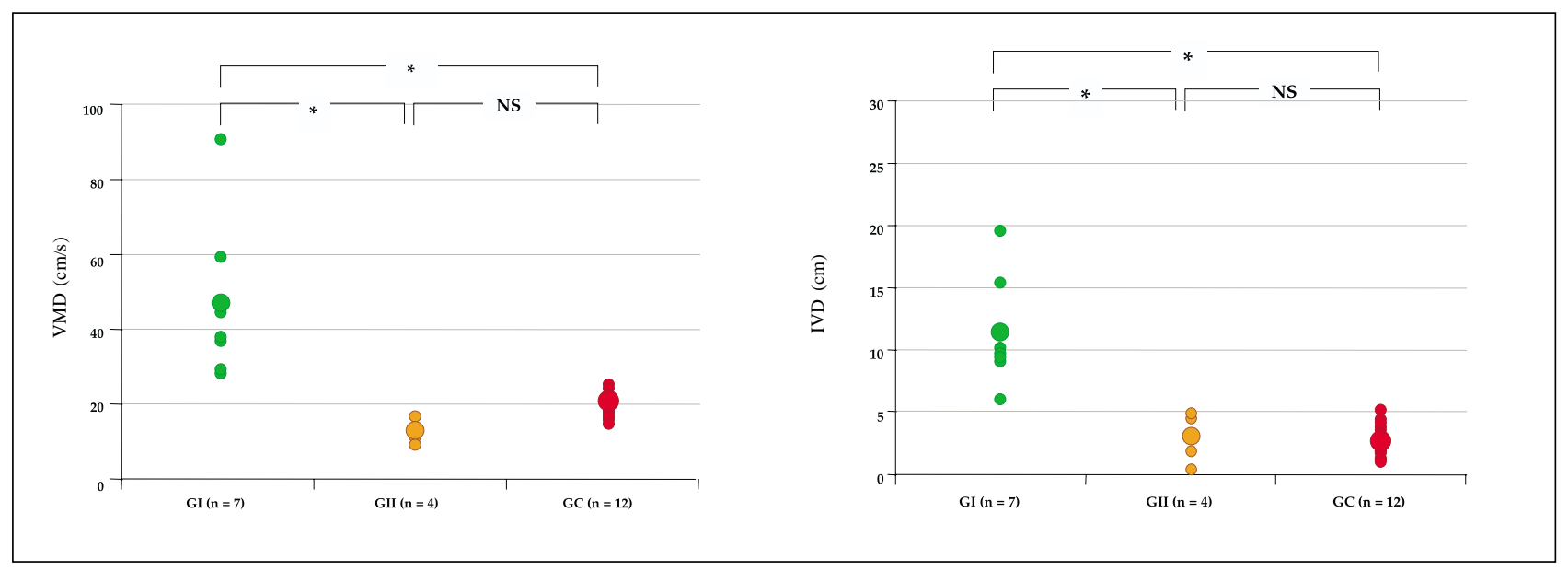

Fig. 5 - Gráficos de distribuição da velocidade máxima de fluxo diastólico (VMD) e integral de velocidade do fluxo diastólico (IVD) no grupo I(GI), grupo II (GII)e grupo controle (GC).

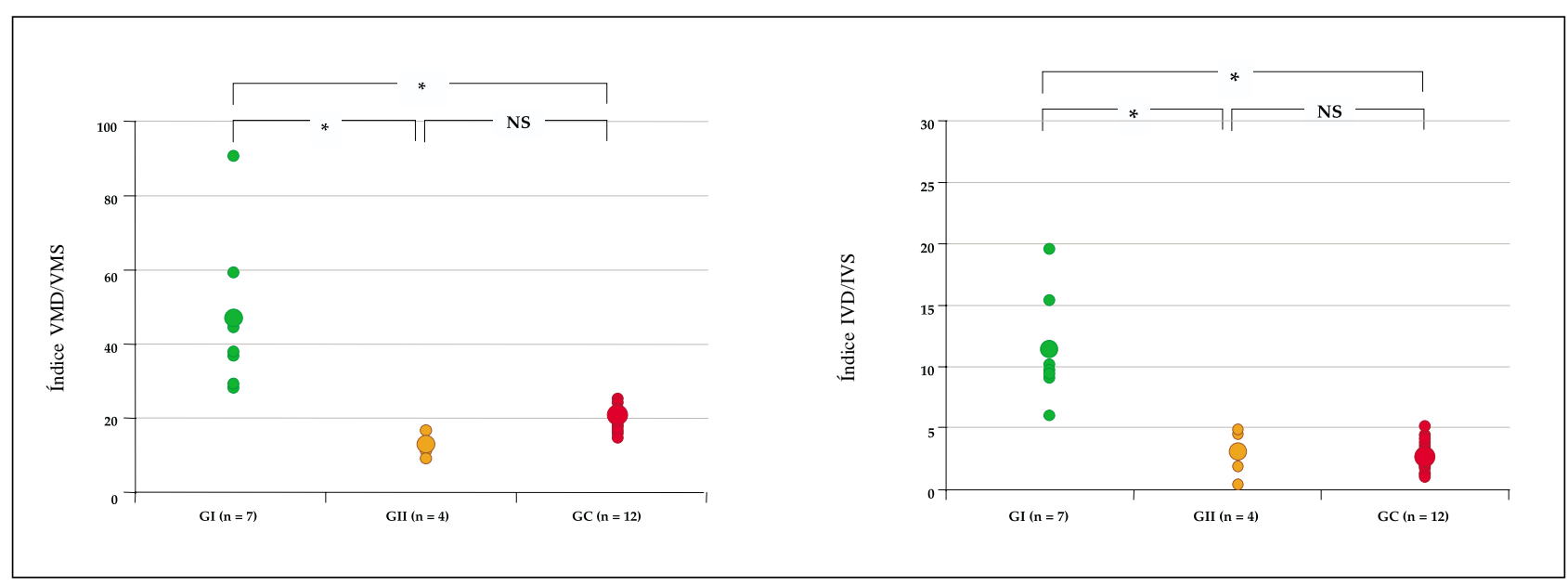

Fig. 6 - Gráficos de distribuição do índice VMD/VMS e IVD/IVS no grupo I (GI), grupo II (GII) e grupo controle (GC).

Analisou-se a distribuição dosíndices VMD/VMSeIVD/ IVS com valores $>0,5$ nos pacientes dos grupos I I II (tab. III).

Observou-se que os índices VMD/VMS e IVD/IVS com valores $>0,5$ ocorreram com predomínio nos pacientes do grupo I em relação ao grupo II.

Ao analisar a $\mathrm{FC}$ dos pacientes no período $\mathrm{PO}$ de revascularização miocárdica pela minitoracotomia constatou-se que os valores médios do grupo I (98bpm) não foram diferentes dos do grupo II (91bpm).

\section{Discussão}

O presente estudo descreve os padrões das curvas espectrais de Doppler de ATIE anastomosada com artéria interventricular anterior pela técnica de minitoracotomia sem CEC. Esta avaliação Ecocardiográfica havia sido realizada previamente em outros estudos com pacientes revascularizados pela técnica cirúrgica convencional com esternotomia mediana e $\mathrm{CEC}^{11-16}$.

Em nosso estudo, a via preferencial paraesternal esquerda foi de fácil manuseio e de rápido aprendizado. Gerou exeqüibilidade de $93 \%$ nos pacientes analisados, superior aos estudos disponíveis na literatura, pela mesma abordagem, em pacientes operados pela técnica convencional ${ }^{11-14}$. Os resultados de nossa casuística, possivelmente, estão relacionados à posição da artéria torácica interna no $\mathrm{PO}$ de revascularização miocárdica pela minitoracotomia, que durante a intervenção cirúrgica, geralmente, nãoé manipulada em sua porção proximal ${ }^{10}$. Permanecendo em seu leito nativo, torna-se mais facilmente acessível a abordagem pelo ECO pela via paraesternal. Em nossa casuística, o único paciente em que não foi possível a avaliação pelo ECO, apresentava má janela acústica nas duas abordagens propostas, em que a anastomose coronária estava pérvia à CINE. Esta dificuldade em abordar a artéria torácica interna por meio do ECO transtorácico não é sinônimo de falência da anastomose, mas sim, uma das limitações do método ${ }^{13}$. O emprego de contrastes ecocardiográficos para otimização das curvas espectrais de fluxo Doppler poderá incrementar sua exeqüibilidade ${ }^{15}$.

Para realização dos estudos Ecocardiográficos, optamos pelos transdutores de $5 \mathrm{MHz}$, por apresentarem menor 
efeito de atenuação tecidual que os de maior freqüência $(7,5 \mathrm{MHz})$, o que pode ter contribuído para a alta exequiibilidade desta abordagem ${ }^{11,12}$. Quando transdutores de 7,5MHz foram utilizados por outros autores, em pacientes revascularizados pela técnica convencional, menores percentagens de visibilização das artérias torácicas internas foram observadas, tanto pela via paraesternal quanto pela supraclavicular ${ }^{13}$.

O número relativamente pequeno de pacientes constituiu uma das limitações do presente estudo. Entretanto, os grupos analisados apresentaram comportamento uniforme nos padrões das curvas obtidos pelo estudo ECO da ATIE anastomosada à interventricular anterior, em relação a sua perviabilidade angiográfica, confirmando assim o valor diagnóstico do método na avaliação funcional não invasiva da anastomose coronária realizada pela técnica de minitoracotomia sem CEC. A ocorrência de obstrução da anastomose coronária no nosso estudo, relacionou-se ao período inicial de aprendizado da Equipe de Cirurgia Cardiovascular com esta nova técnica de revascularização miocárdica. Nesta fase de implementação da técnica, exigiase rigor na avaliação pós-operatória dos resultados, consubstanciado pela associação de métodos propedêuticos invasivos e não invasivos. Destacou-se o fato do ECO ter permitido o diagnóstico precoce de obstrução da anastomose, em fase de PO, período em que a dor precordial pela incisão, bem como alterações eletrocardiográficas ou enzimáticas inerentes a manipulação cirúrgica, dificultam a análise não invasiva dos resultados da intervenção cirúrgica. Vale salientar que não houve caso de óbito ou infarto do miocárdio como conseqüência direta ou indireta da cirurgia de revascularização miocárdica pela técnica de minitoracotomia. $\mathrm{Na}$ atualidade, após a necessária curva de aprendizado da técnica cirúrgica, a experiência sedimentada pela equipe revela baixa incidência de obstrução precoce da anastomose ${ }^{10}$.

Em nossa casuística, observamos que na quase totalidade dos pacientes com anastomoses pérvias à angiografia (grupoI), o predomínio de fluxo diastólico foi o padrão morfológico característico ao estudo Doppler. Achado semelhante foi descrito em estudos que abordaram a mesma anastomose realizada pela técnica cirúrgica convencional ${ }^{12,16}$. Este componente diastólico é considerado o principal determinante do fluxo sangüíneo para o leito coronário, que pode ser passível de alterações na presença de lesões obstrutivas na anastomose arterial ${ }^{12,13,16,17}$.

De fato, em vigência de obstrução desta anastomose, há nítida modificação da morfologia da curva espectral de fluxo da ATIE ao ECO, que passa a ter predomínio sistólico, conforme demonstraram nossos resultados, confirmando achados de outros estudos realizados pela cirurgia convencional. Em apenas um paciente do nosso estudo pertencente ao grupo I, a análise qualitativa e quantitativa de fluxo Doppler evidenciou padrão B (predomínio sistólico) com índices VMD/VMS e IVD/IVS <0,5. Embora a CINE de controle PO deste paciente demonstrasse presença de fluxo angiográfico, através da anastomose, evidenciou lesão estenótica significativa da mesma. Aspecto semelhante de fluxo com predomínio sistólico foi descrito em análises Ecocardiográficos no seguimento tardio de pacientes revascularizados pela técnica convencional. Nesses estudos, o padrão de curva espectral de Doppler com predomínio sistólico ocorreu nos pacientes com estenose $>70 \%$ da luz da artéria torácica interna anastomosada à coronária ${ }^{12,16}$.

No presente estudo, o padrão de morfologia das curvas de fluxo da anastomose dos pacientes pertencentes ao grupo II (padrão B) foi semelhante àquele observado nos indivíduos hígidos do grupo controle, ou seja, de predomínio sistólico. Este componente sistólico do fluxo através da artéria torácica interna anastomosada a uma artéria coronária é responsável pela irrigação de ramos da artéria torácica interna, como as artérias pericardicofrênica, costallateral e tímica ${ }^{16-18}$. A anastomose, uma vez obstruída, perde o elemento de baixa resistência conferido pelo leito coronário durante a diástole, e a artéria torácica interna passa a se comportar como uma artéria não anastomosada.

O amplo fluxo diastólico nos pacientes com anastomoses pérvias justificou o resultado de índices VMD/VMS e IVD/IVS >0,5 presentes nos pacientes do grupo I. O predomínio sistólico nos pacientes com anastomoses obstruídas à CINE (grupo II) refletiu-se no valor dos índices $<0,5$ neste grupo. Outros autores, avaliando o ECO transtorácico em anastomoses realizadas através da revascularização convencional, obtiveram resultados semelhantes ${ }^{12,16}$.

Nossos resultados não foram influenciados por diferentes intervalos de tempo entre intervenção cirúrgica e exames complementares, visto terem sido equivalentes nos dois grupos de pacientes estudados. Tampouco a duração relativa da diástole interferiu nestes resultados, já que não houve diferença estatística de FC dos dois grupos.

Embora útil para o diagnóstico de perviabilidade da anastomose coronária, dúvidas permanecem em relação ao valor do ECO para quantificar diferentes graus de estenose desta anastomose. O uso potencial do método para quantificação da estenose na anastomose coronária, efetuada pela técnica cirúrgica de minitoracotomia, requer futuras pesquisas. Investigações recentes, por meio do ECO transesofágico, têm analisado a reserva de fluxo coronário durante a infusão endovenosa de dipiridamol ${ }^{19}$ ou adenosina ${ }^{20}$ que atuam nos vasos de resistência da circulação coronária. É possível que estudos prospectivos com CINE quantitativa e avaliação do comportamento do fluxo pela artéria torácica interna anastomosada à coronária com o uso do ECO transtorácico, com estresse farmacológico induzido por vasodilatadores, possam abrir novas perspectivas para o esclarecimento desta questão.

O presente estudo confirma a utilidade do ECO como método não invasivo efetivo para o diagnóstico precoce $\mathrm{PO}$ do estado funcional da anastomose da ATIE com a artéria interventricular anterior e amplia as aplicações práticas do método na avaliação do resultado cirúrgico nas diversas formas de revascularização miocárdica disponíveis. Além disso, acena com a possibilidade do seu uso na avaliação pós-operatória tardia durante o acompanhamento longitudinal dos pacientes revascularizados. 


\section{Referências}

1. Levy RI - The decline in cardiovascular disease mortality. Ann Rev Public Health 1981; 2: 49-70.

2. Marmot MG - Interpration of trends in coronary heart disease mortality. Acta Med Scand 1985; 701(suppl): 58-65.

3. Mestres CA, Rives A, Igual A, Vehi C, Murtra M - Atherosclerosis of the internal mammary artery. Histopathological analysis and inplications on its results in coronary artery bypass graft surgery. Thorac Cardiovasc Surgeon 1986; 34: 356-8.

4. Bennetti FJ, Nasseli G, Wood M, Geffner L-Direct myocardial revascularization without extracorporeal circulation. Experience in 700 patients. Chest 1991; 100: 312-6.

5. Buffolo E, Andrade JCS, Branco JN, Teles CA, Aguiar LF, Gomes WJ - Coronary artery bypass grafting without cardiopulmonary bypass. Ann Thorac Surg 1996; 61: 63-6.

6. Sharp TG - Editorial Comment: minimally invasive coronary bypass surgery. ACC Current Journal Review 1996; May/June: 63-4.

7. Acuff TE, Landreneau RJ, Griffith BP, Mack MJ - Minimally invasive coronary artery bypass grafting. Ann Thorac Surg 1996; 61: 135-7.

8. Schaff HV, Cable DG, Rihal CS, Daly RC, Orszulak TA - Minimal Thoracotomy for coronary artery bypass: Value of imediate post procedure graft angiography. Circulation 1996; 94(suppl I): 51.

9. Jatene FB, Fernandes PMP, Stolf NAG et al - Cirurgia de revascularização do miocárdio minimamente invasiva com utilização de videotoracoscopia. Arq Bras Cardiol 1997; 68: 107-11.

10. Teles CA, Buffolo E, Petrizzo A, Ribeiro E, Silva L, Mathias W-Revascularização do miocárdio minimamente invasiva. Rev Bras Cir Cardiovasc 1996; 11: 82-5.

11. Takagi T, Yoshikawa J, Yoshida K, Akasaka T, Maeda K - Nonivasive measurement of left internal mammary Doppler of left internal mammary artery bypass graft flow by Duplex Doppler echocardiography from the supraclavicular fossa. J Am Soc Echocardiogr 1993; 6: 347-81.
12. Crowley JJ, Shapiro LM - Noninvasive assesment of left internal mammary artery graft patency using transthoracic echocardigraphy. Circulation 1995; 92 : 25-30.

13. De Bono P, Samani NJ, Spyt TJ, Hartshorne T, Thrush AJ, Evans DH - Transcutaneous ultrasound measurement of blood-flow in internal mammary artery to coronary artery grafts. The Lancet 1992; 339: 379-81.

14. Krinje R, Lyttwin RM, Hötgen R, Heinrich KW - Doppler sonographic examination of the internal mammary artery graft: Comparison of supraclavicular and high intercostal signals. Cardiology 1993; 82: 48-50.

15. Munvagh S, Foley D, Aeschbacher BC, Klarich KK, Seward JB - Second Harmonic imaging of an intravenously administered echocardiographic contrast agent. J Am Coll Cardiol 1996; 27: 1519-25.

16. Nasu M, Takagi T, Akasaka T et al - Non-invasive Doppler techinique for detection of flow velocity in left internal mammary artery grafts. Cardiov Surg 1994; 2 : 207-11.

17. Van der Meulen J, Van Son JAM, Van Asten WNJC, Skotnick SH, Laquet LK - Intraoperative Doppler spectrum analysis of blood flow in the internal mammary artery used for myocardial revascularization. Thorac Cardiovasc Surg 1991; 39: 281-3.

18. Brandyk DF, Galbraith TA, Haasler GB, Almassi H-Blood flow velocity of internal mammary artery and saphenous vein grafts to the coronary arteries. J Surg Res 1988; 44: 342-51.

19. Iliceto S, Marangelli V, Memmola C, Rizzon P - Transesophageal Doppler echocardiography evaluation of coronary blood flow velocity in baseline conditions and during dipyridamole-induced coronary vasodilation. Circulation 1991; 83: 61-9.

20. Redberg RF, Sobol Y, Chou TM et al - Adenosine-induced coronary vasodilatation during transeophageal Doppler echocardiography. Circulation 1995; 92 190-6. 